\title{
GEOMETRIC SYMMETRY TECHNIQUES FOR PARTIAL DIFFERENTIAL EQUATIONS
}

\author{
NAGHMANA TEHSEEN
}

(Received 7 October 2015; first published online 11 December 2015)

2010 Mathematics subject classification: primary 35A30; secondary 58A17, 58A15, 58A10, 58A20, 58A30, 58J72, 35G20, 35K25, 35K59, 35Q53.

Keywords and phrases: Vessiot theory, Frobenius integrability, Pfaffian system, solvable structures, geometric partial differential equations, Shannon entropy, evolution equations.

The thesis applies Vessiot theory and exterior calculus to find local solutions of partial differential equations (PDEs). We examine the classes of PDEs which are represented by vector fields forming the Vessiot distribution. In general the Vessiot distribution is not Frobenius integrable. We consider the problem of computing the integrable subdistributions of the nonintegrable Vessiot distribution for PDEs. We develop and apply a method to find the largest integrable sub-distributions and hence (local) solutions of the PDEs. A more general class of symmetries known as solvable symmetry structures plays an important role in our work. A novel feature of our approach is the formulation of the 'quasi-group invariance method'. In particular, we show how the solvable symmetry structure of the original PDEs can be used to construct integrable subdistributions leading to invariant solutions of the PDEs. We also extend this result to higher dimensional PDEs. We give new results concerning the Frobenius integrability and solution of evolution equations admitting travelling wave solutions. We also discuss 'local' conservation laws for evolution equations. Some consideration of the application of this technique to analyse Shannon entropy of evolution equations is taken at the end.

Each chapter contains an outline of its structure. Chapters 3 and 4 appeared as [4]. The results in Chapter 5 have appeared in [2]. The results in Chapter 6 have appeared in [1]. Chapter 8 has been published in [3].

We begin Chapter 2 by reviewing some preliminary material, that is, essentially an introduction to the basic concepts of calculus on manifolds, symmetries and representation of differential equations as an exterior differential system.

Thesis submitted to La Trobe University in November 2014; degree approved on 20 May 2015; principal supervisor Geoff Prince; cosupervisor Philip Broadbridge.

(c) 2015 Australian Mathematical Publishing Association Inc. 0004-9727/2015 \$16.00 
In Chapter 3, we give an introductory explanation of the procedure of reduction of order, which includes a geometric representation of an integrating factor for ordinary differential equations (ODEs) which leads to first integrals. This reduction of order technique deals with Frobenius integrable distributions. However, our work is dealing with nonintegrable distributions. We give a four-step method which begins by considering the problem of locating the integrable sub-distributions of a nonintegrable distribution. In addition, since we are dealing with Frobenius integrability and symmetries, we also give various new results concerning the Frobenius integrability of a distribution.

In Chapter 4, we introduce the idea of the Vessiot distribution for the second order PDEs in one dependent variable and two independent variables of the form

$$
u_{y y}=F\left(x y, u, u_{x}, u_{y}, u_{x x}, u_{x y}\right) .
$$

In general, the Vessiot distribution is not Frobenius integrable. If a distribution is not Frobenius integrable, then it is interesting to ask for sub-distributions which are Frobenius integrable. Vessiot has given an existence theory of such Frobenius integrable sub-distributions. We consider the problem of computing the integrable sub-distributions of the nonintegrable Vessiot distribution. The main new result that we present is the application of the four-step method of Chapter 3. We establish that we can use this method to find integrable sub-distributions of the Vessiot distribution which satisfy an appropriate independence condition and represent local solutions of the PDE system. We show how to use a solvable symmetry structure to integrate a PDE in original coordinates but, most importantly, we show how to impose a solvable structure on a PDE to find integrable sub-distributions of the Vessiot distribution. We call this the 'quasi-group invariance method'. We also produce an energy dispersive (EDS) method to solve the differential conditions in step two of the method.

We discuss the utility of our technique and also present an application of our technique to finding the additively separable solutions for evolution equations. We conclude this chapter with an observation about the relationship between Darboux invariants and the extra 1-forms in our method for second order hyperbolic partial differential equations in two independent variables and one dependent variable. For each of the cases we give a concrete example.

In Chapter 5, we enlarge the scope of applicability of our technique by allowing more independent and dependent variables into the picture. We apply the geometric technique to find the largest integrable sub-distributions of the Vessiot distribution of the multi-dimensional second order PDEs. The most significant new result in this chapter is the application of our method to second order PDEs in more than two independent variables. This is because the usual Lie reduction process of such a PDE could lead to another PDE in two independent variables. However, our method reduces the problem of describing the solutions of the Lie-reduced PDE to the Frobenius theorem, which is an ODE problem and is not generally possible within the standard Lie approach. 
Chapter 6 presents an application of our technique to find travelling wave solutions of evolution PDEs. The aim is to give a new geometric perspective on these solutions, viewed as the projection to the configuration space of the common level sets of functions on a jet bundle. In particular, these common level sets are the integral manifolds of the Vessiot distribution. We give some new results concerning the Frobenius integrability and solution of evolution equations admitting travelling wave solutions. In particular, we give a powerful result which explains the extraordinary integrability of some of these equations. We also discuss 'local' conservation laws for evolution equations in general and demonstrate all the results for the Korteweg de Vries equation.

In Chapter 7, we discuss another new application of our technique: the analysis of Shannon entropy of second order evolution equations on solution surfaces.

Chapter 8 is devoted to study the monotonicity of Shannon entropy of fourth order diffusion equations. Although all second order nonlinear diffusion equations imply monotonically increasing Shannon entropy, this is the exception rather than the rule for fourth order diffusion equations. By a simple local analysis of the reaction-diffusion equation for entropy, we discuss a class of such information-irreversible equations with nonlinear diffusivity and for which entropy density increases everywhere on the real line, except in special equilibrium states.

Finally, in Chapter 9 we present the conclusions of the thesis and a list of possible future directions.

\section{References}

[1] G. Prince and N. Tehseen, 'Evolution equations: Frobenius integrability, conservation laws and travelling waves', J. Phys. A 48(40) (2015), 405205-405213.

[2] N. Tehseen, 'Explicit solutions of PDE via Vessiot theory and solvable structures', J. Phys. A 47(31) (2014), 315207-315225.

[3] N. Tehseen and P. Broadbridge, 'Fourth order diffusion equations with increasing entropy', Entropy 14(7) (2012), 1127-1139.

[4] N. Tehseen and G. Prince, 'Integration of PDEs by differential geometric means', J. Phys. A 46(10) (2013), 105201-105220.

NAGHMANA TEHSEEN, Department of Mathematics and Statistics,

La Trobe University, Victoria 3086, Australia

e-mail: naghmanatehseen@gmail.com 